\title{
Quasi "una cosa sola": Ragazza che precipita di Dino Buzzati fra racconto e immagine dipinta
}

\author{
Paolo Senna \\ Università Cattolica del Sacro Cuore, Biblioteca d'Ateneo \\ paolo.senna@unicatt.it
}

\section{Riassunto}

La relazione tra parola e immagine in Buzzati si esprime con i tratti di un marcato polimorfismo che prevede un ventaglio cospicuo di possibilità realizzative: da parole o blocchi di testo inseriti in quadri e disegni, a veri e propri rifacimenti in pittura di nuclei narrativi omogenei precedentemente trattati in un testo scritto o, viceversa, rielaborazioni in chiave narrativa di immagini preesistenti. Tra i diversi casi di tali "riscritture" o "rifacimenti", Ragazza che precipita appare significativo per osservare da vicino l'officina buzzatiana. Al racconto, uscito in prima istanza sul Corriere della Sera nel 1960 (e da qui ripreso in Il colombre nel 1966), Buzzati fece seguire nel 1962 un dipinto dal medesimo titolo. L'articolo presenta un'analisi sinottica delle due realizzazioni evidenziandone le sovrapposizioni e i significativi scostamenti, ponendo in evidenza i valori costitutivi e i richiami intertestuali. Parole chiave: Dino Buzzati; Giacomo Leopardi; Ragazza che precipita; parola e immagine; giovinezza.

Abstract. Almost "one thing": Dino Buzzati's Ragazza che precipita between story and
painted image

The relationship between word and image in Buzzati's works is revealed with a conspicuous range of possibilities: words or text blocks included in paintings and drawings, real remakes in painting of previously written stories, and, vice versa, reworking in a narrative key of pre-existing images. Among these "remakes", Ragazza che precipita can show closely the Buzzati's workshop. The story, first published in the Corriere della Sera newspaper in 1960, inspired a painting in 1962. The article presents a synoptic analysis of the two works, highlighting the intertextual references and the important differences.

Keywords: Dino Buzzati; Giacomo Leopardi; Ragazza che precipita; text and image; youth. 
Il fatto è questo: io mi trovo vittima di un crudele equivoco. Sono un pittore il quale, per hobby, durante un periodo purtroppo alquanto prolungato, ha fatto anche lo scrittore e il giornalista. Ma dipingere e scrivere per me sono in fondo la stessa cosa. Che dipinga o che scriva, io perseguo il medesimo scopo, che è quello di raccontare delle storie (Buzzati 1958, 2013, p. 143).

Il passo, giustamente celebre e rievocato con molta frequenza dagli studiosi, rende ragione di quella intercambiabilità e quella complementarità fra parola e immagine, già accuratamente rilevate da Nella Giannetto (1998), che costituiscono carattere specifico dell'opera di Dino Buzzati. Lo stretto legame tra le due modalità espressive è infatti radicato nell'autore del Deserto dei Tartari fin dalle prime opere e, a ben vedere, fin dai più antichi esercizi di scrittura: così è per le lettere scambiate con l'amico Arturo Brambilla dove il connubio tra parola e immagine include il gioco, tra il serio e il faceto, esemplato sui geroglifici egizi; così è anche per le precoci illustrazioni per The Haunted Palace di Poe dotate di didascalie; così è, ancora, per le missive indirizzate alla giovane amata Bibi (Beatrice) Giacometti, sulle quali campeggiano illustrazioni quando non veri e propri racconti figurati; così è infine nelle stesure manoscritte delle opere buzzatiane, in cui il processo narrativo si avvale di numerosi disegni con i quali lo scrittore dà forma ai propri protagonisti e delinea i profili dei paesaggi. Per comprendere appieno tale rapporto e quindi la collaborazione poietica tra parola e immagine sarebbe utile, dove possibile, poter disporre di riproduzioni facsimilari degli autografi e questo non tanto per le opere che rendono manifesta la collaborazione tra parola e immagine al termine del processo editoriale (è il caso di La famosa invasione degli orsi in Sicilia e Poema a fumetti), ma in particolare per tutte le altre che la innescano durante il percorso creativo, nonostante l'esito a stampa - e non sempre per volontà dell'autore - risulti in definitiva veicolato su un singolo canale espressivo. ${ }^{1} \mathrm{E}$ ciò che è stato recentemente proposto per Il reggimento parte all'alba (Buzzati, 2018), che Lorenzo Viganò ha inteso pubblicare unitamente a una scelta delle pagine originali dall'agenda manoscritta di Buzzati, in modo tale da ripristinare anche per il lettore quel dialogo muto e fecondo tra parola e immagine che ha fatto parte, e parte rilevante, dell'iter creativo.

Dino Buzzati è perciò da sempre scrittore visivo oltre che, come è stato osservato in più occasioni, scrittore visionario. A sostegno di questa affermazione confortano le parole che l'autore in prima persona ha espresso in diverse circostanze e che, nel dettaglio, traggo da una pubblicazione poco nota. Si tratta di un dibattito con Ferdinando Giannessi tenutosi presso l'Associazione culturale "L'argine", che portò lo scrittore il $1^{\circ}$ giugno 1970 ad Ostiglia, in

1. Nella Giannetto (2001, p. 19) ricorda che Bàrnabo delle montagne "nasce corredato di disegni". Allo stesso modo Lorenzo Viganò (2006, p. 18) rammenta che sia in Bàrnabo che nel Segreto del Bosco Vecchio "Buzzati 'semina' il testo di piccole illustrazioni (quadretti, a volte usati come capilettera, per visualizzare situazioni, paesaggi, personaggi) che però non verranno mai pubblicati. Non si sa se questa scelta abbia mortificato Dino Buzzati, probabilmente sì, soprattutto quando Orio Vergani gli consigliò di togliere i disegni dal Segreto del Bosco Vecchio perché ricordavano troppo Yambo". 
provincia di Mantova, per un incontro pubblico. Di questa comunicazione orale rimane traccia nella trascrizione stampata in un raro opuscolo nelle cui pagine Buzzati dichiara a proposito di Poema a fumetti:

Non è che io abbia avuto la intenzione di fare precisamente un libro a fumetti veri e propri [...]. A me è piaciuto fare un libro di disegni intercalati con il testo, in cui il testo e i disegni facessero una cosa sola, per riassumere, in un certo senso, tutte le atmosfere, gli ambienti, le situazioni che io ho massimamente amato nella mia vita; [...] Ė una cosa che ho fatto perché mi piaceva farla e d'altra parte non è una mia invenzione di tarda età, perché io lo stesso lavoro, se si può dire così, lo facevo quand'ero ragazzo. Da ragazzo, facevo delle storie, come tanti ragazzi del resto, o dei poemi più o meno sballati e li decoravo, li accompagnavo sempre con dei disegni di modo che questa voglia di mettere insieme l'immagine e la parola è sempre stata un mio desiderio (Buzzati, 1971, p. 5).

Testo e disegni fanno "una cosa sola". Se questa affermazione è vera per Poema a fumetti, e se è altrettanto vero che il disegno in Buzzati è una opzione che accompagna in misura costante il percorso creativo, è possibile estendere tale concetto all'opera intera di Buzzati, seppur con le dovute distinzioni che tengano conto dell'evoluzione di stile e mezzi nel corso del tempo. La relazione tra parola e immagine in Buzzati si dispiega con i tratti di un marcato polimorfismo (per cui cfr. Giannetto, 1998, 2001), e si incanala secondo percorsi originali che vanno ben oltre il semplice rapporto, che potremmo dire di grado zero, di affinità di stile nell'impiego di due canali comunicativi differenti. $^{2}$ Assistiamo infatti a una molteplicità di corrispondenze che prendono in considerazione tanto la coesistenza autonoma dei due diversi moduli mediali quanto l'incorporazione di figure dipinte o disegnate e blocchi di testo più o meno ampi e legati fra loro in un unico supporto. Un ventaglio di possibilità che richiederebbe uno spoglio sistematico finalizzato a produrre una plausibile tassonomia critica complessiva dell'opera. Molto più modestamente, mi limito qui a proporre un tentativo di indicizzazione, utile almeno come ipotesi di lavoro:

1. In primo luogo vi sono i titoli e le didascalie presenti su quadri e disegni; intese, queste ultime, non come "semplice spiegazione", ma come vero e proprio "commento [...] battuta aforistica allusiva che prende spunto dall'immagine per sovrapporvi nuovi significati” (Giannetto, 2001, p. 18). La didascalia si propone come elemento non definitorio, ma polisenso, anche con funzione ironica o di depistaggio, e mira a produrre uno scarto sensibile tra l'oggetto rappresentato e la sua interpretazione allo scopo di sottolineare possibili valori ulteriori, giocando sull'equivoco metaforico e sullo slittamento metonimico. Tale risultato è spesso ottenuto anche dal solo titolo, per sua natura assai più

2. Quali possono essere, in termini generali (e quindi senza riferimento specifico allo stile di Buzzati), un linguaggio espressionista o impressionista tanto nella scrittura quanto nella pittura. 
sintetico della didascalia, nel suo porsi come "soglia" polivalente e enigmatica alla fruizione dell'immagine.

2. Vi sono poi le parole inserite materialmente nei dipinti con l'intento: (a) di proporre le battute e i discorsi dei personaggi, secondo una tecnica molto prossima a quella del fumetto; oppure (b) di suggerire il flusso della narrazione, quasi che ci trovassimo davanti a fotogrammi successivi o a rappresentazioni contemporanee di realtà e oggetti diversi. Il dipinto può anche risultare diviso in più settori che suggeriscono allo spettatore/lettore la dimensione temporale. Caso limite di questo tipo è Rebus. La morta nel solaio (1958), dove l'elemento verbale (la lettera "l" maiuscola e minuscola) si fa a tal punto ambiguo da essere considerato anche per il suo mero valore grafico, quasi fosse esso stesso un'immagine.

3. Altro caso è rappresentato dalle composizioni che nascono con la cooperazione iterata di parola e immagine, come Poema a fumetti e i Miracoli di Val Morel. In queste opere la relazione tra parola e immagine non si esaurisce nella singola tavola o nello spazio dove avviene la loro immediata interazione ma, essendo inserite in un "testo" di più vasto respiro o paginazione, a tale rapporto si aggiunge l'interdipendenza fra le diverse tavole o pagine nel loro costituirsi come una narrazione unica e prolungata, ossia come "libro".

4. Situazione particolare è data infine da quelli che potremmo definire rifacimenti o riscritture parallele. Ciò avviene sia (a) tra immagine ed immagine, secondo un programma che prevede tanto l'imitazione o la citazione di immagini altrui quanto l'autocitazione di esperienze pregresse: basti pensare alla ripresa in Poema a fumetti di molti soggetti già apparsi in dipinti buzzatiani precedenti, come Il Babau o Le mille e una notte (cfr. Ferrari, 2006, pp. 70-83, Buzzati, 1971, p. 5); oppure (b) tra immagine e parola, come nel caso dei quadri nati da racconti, in un gioco continuo che punta a fondere e confondere testo e immagine, assumendo in un'ottica visiva quanto precedentemente era stato esposto con il veicolo della parola scritta. Ė questo il caso di alcuni racconti o narrazioni più distese - come Ragazza che precipita (1960), Il colombre (1961), Un amore (1963), Il Babau (1967) - il cui soggetto viene ripreso e trasposto in pittura, dando vita ai dipinti, spesso omonimi, Ragazza che precipita (1962), Il colombre (1970), Un amore (1965) e Laide (1967), e infine a Il Babau (1967). Sembrerebbe quindi che vi sia originariamente un testo scritto al quale Buzzati intende successivamente riservare una 'traduzione' in immagine visiva. Tuttavia la relazione fra questi esiti non si esprime esclusivamente secondo l'univoca direzione scrittura $\rightarrow$ pittura, al punto che diversi studiosi hanno espresso più di un dubbio sul rapporto cronologico esistente tra le diverse esecuzioni. Il caso di Toc Toc appare al proposito emblematico. Il tema ha una realizzazione pittorica nel 1956 dove viene presentato uno spaventoso cane nero, che raffigura la morte, mentre bussa alla porta di un edificio a più piani. Le brevi battute incorporate nel dipinto vengono riadattate con qualche modifica nel brevissimo racconto Il busso della porta (Buzzati, 2007, p. 386) apparso insieme ad altri rapidi sketch con il titolo cumulativo Progressioni sul Corriere della Sera il 3 ottobre 1965 e successivamente ristampato in volume 
in Il colombre nel 1966. Il tema però, anche se con taglio e situazione diversi, aveva già fatto la sua apparizione in Eppure battono alla porta e, con toni più sinistri, in Il dolore notturno, entrambi inclusi in I sette messaggeri del $1942 .{ }^{3}$ Racconto dipinto o pittura narrata? Come si vede, esiste in Buzzati un nucleo narrativo omogeneo che viene trattato in modalità e linguaggi differenti che prevedono non solo inevitabili varianti, ma anche la reiterazione a lungo termine del medesimo tema, perfino in anni (e decenni) diversi. Si dà certamente il caso di racconti che vengono successivamente reinterpretati con il linguaggio pittorico (scrittura $\rightarrow$ pittura), ma esiste anche la circostanza opposta, cioè di immagini disegnate o dipinte che vengono successivamente manipolate e diluite nel linguaggio verbale (pittura $\rightarrow$ scrittura), attuando strategie ecfrastiche complesse. Questi nuclei narrativi possono dunque essere più volte ripresi e continuamente reiterati secondo un gioco di rimaneggiamenti e riferimenti progressivi che conferiscono speciale compattezza e coesione all'opera buzzatiana. La relazione fra parola e immagine è quindi estremamente articolata $\mathrm{e}$ deve tenere conto di questa possibilità continua di rielaborazione e interpolazione che costituisce la cifra speciale dello stile dell'autore. ${ }^{4}$

In questi casi di rifacimenti o riscritture parallele viene stabilita una speciale modalità di relazione in cui, per esplicita volontà autoriale, racconto e immagine si collocano a un pari livello di autonomia realizzativa e, al contempo, instaurano tra loro corrispondenze eteronomiche, portatrici di significative evoluzioni informative. Nel momento di ritorno sul tema precedentemente trattato si assiste infatti a un gamma di interventi che vanno dalla lineare rielaborazione in un nuovo linguaggio, al cambio del punto di vista, fino a un ripensamento del fuoco del tema, del quale l'autore sceglie di evidenziare aspetti diversi, o alla proposta di una conclusione discorde. Se osservati in sinossi, perciò, i due "testi" possono mostrare differenze anche consistenti, proprio in virtù degli scarti che si vengono a produrre tra l'una e l'altra realizzazione del medesimo soggetto. Si tratterà quindi di un'interazione non rettilinea né perfettamente sovrapponibile, ma diffratta, che amplifica le potenzialità

3. Il dolore notturno è racconto degno di interesse anche nell'ottica della relazione parolaimmagine. Nelle sue pagine è descritto un inquietante disegno con "schegge di assurde immagini in cui prendevano forma figurazioni di occhi, si intrecciavano in ridda, e, a osservarli lungamente, si vedevano ruotare gli uni entro gli altri, di un moto che pareva eterno" (Buzzati, 2006, p. 179). Disegno che però può essere visto solo da uno dei due fratelli protagonisti del racconto, mentre all'altro il foglio appare misteriosamente bianco.

4. In Buzzati assistiamo quindi alla realizzazione, individuata da Mitchell, sia di un imagetext, ovvero un oggetto compatto in cui ci si trova in compresenza di parola e immagine, sia di un image-text inteso come estrema possibilità delle relazioni fra immagine e testo (cfr. Mitchell, 1994). La discussione teorica sui rapporti tra parola e immagine è vastissima. Con l'intento di tracciare le linee di un fecondo dibattito in essere mi limito a segnalare i lavori teorici di Boehm (2009), Mitchell (1994, 2017, 2018) e Cometa (2004, 2012). Per ciò che riguarda i rapporti tra letteratura italiana e linguaggi visivi rimando, per motivi di sintesi, ai contributi inclusi in Spigoli (Ed.) (2018) e alla bibliografia ivi indicata, mentre per specifiche attinenze alle opere di Buzzati si vedano: Caspar (1979, 2001), Crotti (1977), Dal Mas \& Endrizzi (1998), Giannetto (1998, 2001), Laganà Gion (1982), Piccolo (2011), Toscani (1987), Zucco (1997). 
dell'opera e ne rende manifeste le varie sfumature di significati. In quest'ottica un testo come Ragazza che precipita può rivelarsi particolarmente utile per osservare da vicino le scelte stilistiche buzzatiane.

Il racconto Ragazza che precipita esce per la prima volta sul Corriere della Sera il 16 marzo 1960 (Buzzati, 1960), per essere poi ripreso in Il colombre (Buzzati, 1966, 2007) e successivamente nella Boutique del mistero (Buzzati, 1968). Nel passaggio all'edizione in volume il testo rimane sostanzialmente inalterato, dal momento che si registra una sola piccola modifica apportata dall'autore. ${ }^{5}$ Nel frattempo, nel 1962, lo stesso soggetto è ripreso in un quadro dal medesimo titolo (tempera su tela, dimensioni $70 \times 100$, collezione privata; l'immagine è riprodotta in coda al presente lavoro). L'elaborazione di questo tema si colloca dunque nel pieno degli anni Sessanta quando sia nella produzione narrativa che in quella pittorica Buzzati lascia ampio spazio alla figura femminile (Giannetto, 2001, p. 21). Più che di una illustrazione vera e propria del testo scritto (cfr. Giannetto, 1998, p. 597) il raffronto fra le due opere - lo anticipo fin d'ora - suggerisce in questo caso un differente trattamento del nucleo narrativo che presenta non solo divergenze veicolari (ossia da testo scritto a immagine), ma anche sostanziali discordanze in merito allo sviluppo della vicenda.

Nel racconto è narrata la storia di Marta, una ragazza di diciannove anni che, giunta alla sommità di un grattacielo altissimo (superiore ai cinquecento piani), si lascia cadere. Si sta facendo sera e il cielo diffonde la luce soffusa del tramonto mentre la città si riempie a poco a poco di un brulichio sempre più fitto e vivace di luci. Voli di questo genere non sono rari da questo grattacielo che proprio a motivo di tale originale "spettacolo" giustifica i prezzi straordinariamente elevati dei suoi appartamenti. Marta cade inesorabilmente, mentre nei piani alti gente vestita alla moda osserva il suo volo e le rivolge la parola, chiedendole di fermarsi almeno per il tempo di un drink o addirittura cercando di afferrarla. La ragazza ha fretta d'arrivare in basso, verso una plausibile festa che sembra apparecchiarsi appositamente per lei. Il tempo della caduta è eccezionalmente dilatato, proprio per la prodigiosa altezza del palazzo e così la ragazza ha modo di riflettere e di guardarsi intorno, osservando che altre giovani donne stanno compiendo il suo stesso folle volo. Man mano che discende verso i piani più bassi si rende conto che anche la notte si sta concludendo e a poco a poco inizia a farsi giorno. Precipitando verso terra, Marta si accorge che molto probabilmente non riuscirà ad arrivare in orario per quella festa alla quale invece desiderava essere protagonista: il tempo a sua disposizione si è consumato nella dissennata discesa e la festa rimarrà un appuntamento mancato. La battuta finale è lasciata a una coppia in un interno borghese il

5. Si tratta di una semplice soppressione dell'avverbio "ancora" nella frase: "Certo la distanza che la separava ancora dal fondo, cioè dal livello delle strade, era immensa". Altri interventi sono di natura esclusivamente grafica e consistono nella trascrizione in tondo di elementi lessicali stranieri tipograficamente resi in corsivo nella stampa sul Corriere della Sera ("tabarins", "cocktails", "chic", "drink"). 
mattino successivo (Buzzati è preciso: sono le "nove meno un quarto") quando moglie e marito osservano il momento conclusivo della caduta di Marta che ha ormai assunto le sembianze di "una vecchia. [...] Una vecchia decrepita" (Buzzati, 2007, p. 310).

Il volo di Marta è immagine della vita: osservata con travolgente desiderio nell'età della giovinezza, si consuma rapidamente per raggiungere uno scopo, per soddisfare un bisogno di felicità che non trova esito se non nella morte, lasciando inespresso tutto quel potenziale di energie e positività che la giovinezza sembrava invece promettere. Marta è dunque la ragazza che precipita, non solo e non tanto perché affonda velocemente verso la propria distruzione, ma perché si affanna, affretta (ovvero, precipita) le sue scelte consumandosi nella attesa vana di un evento al quale non saprà arrivare, segno di una improduttiva speranza. Il tema si riallaccia a uno dei concetti chiave di Buzzati, ovvero l'attesa o piuttosto l'aspettativa di una realizzazione che non giunge a compimento, la consapevolezza di ritrovarsi in ritardo, quasi segnati da una fondamentale asincronia esistenziale che rende impossibile conquistare la vera pienezza. Un motivo così radicato al punto che la caduta di Marta, spinta da una accelerazione di gravità superiore a ogni forza umana, ricorda da vicino quella stessa "comune forza" che "trascina nel gorgo" di Un amore, romanzo in elaborazione proprio nei primi anni Sessanta (Buzzati, 1963); e, ben più addietro, rammenta anche nel lessico citato quasi alla lettera quella "precipitazione di chi teme d'arrivare in ritardo" narrata in Vecchio facocero (Buzzati, 2006, p. 214): così è Marta che si precipita inutilmente per non arrivare tardi all'appuntamento con la vita.

Il nucleo narrativo si fonda su una radice leopardiana. Il poeta di Recanati è stato molto amato da Buzzati tanto che la sua prosa appare fitta di venature che ne richiamano temi e stile (Sandrini, 2001, Lazzarin, 2005). Stefano Lazzarin ha dimostrato, osservando segnatamente la narrativa breve, come la frequenza di un lessico di matrice leopardiana fortemente caratterizzato da evocatività (quello che Luigi Blasucci ha efficacemente definito "segnale dell'infinito", cfr. Blasucci, 1985), faccia sistema con la ricorrenza di meccanismi di accumulazione, già tipici della poetica di Leopardi. ${ }^{6}$ Il caso di Ragazza che precipita, sebbene non sia stato considerato da Lazzarin (2005), appare di interesse al punto da spingere a qualche approfondimento.

Il racconto ha per protagonista una giovane in attesa di partecipare a una "festa [...] che sognava da quando era bambina" (Buzzati, 2007, p. 309). Marta sta vivendo quella stagione in cui, secondo Leopardi, "s'aspetta / bramosamente il dì festivo" (La sera del dì di festa, vv. 40-41), quell'"età fiorita [...] come un giorno d'allegrezza pieno, / giorno chiaro, sereno, / che precorre alla

6. "L'evocatività buzzatiana appare fondata consapevolmente sull'esempio di Leopardi e anzi, come vedremo, si direbbe che i meccanismi evocativo-suggestivi della prosa buzzatiana siano ricalcati su quelli che funzionavano così bene nel contesto lirico dei Canti. Tutto questo significa, fra l'altro, che Leopardi rappresentò per Buzzati innanzitutto un modello di stile" (Lazzarin, 2005, p. 34; corsivo nel testo). 
festa di tua vita" (Il sabato del villaggio, vv. 44-47); l'età in cui, per l'appunto, ci si veste "a festa", ci si ammira vicendevolmente e si gode degli sguardi altrui ("Tutta vestita a festa / la gioventù del loco / lascia le case, e per le vie si spande; / e mira ed è mirata, e in cor s'allegra", Il passero solitario, vv. 32-35). Allo stesso modo Marta è abbigliata con un "modesto abito" che però la luce del tramonto esalta, "rendendolo chic" e facendola sentire interessante (Buzzati, 2007, p. 306-307). Non mi dilungherò per ovvi motivi sul significato della festa, e della sua attesa, come emblema in Leopardi del raggiungimento della felicità e della speranza di raggiungerla. Ma è significativo rilevare come l'idea di festeggiamenti e spettacoli pubblici faccia capolino nello Zibaldone, opera che sappiamo essere stata ben nota a Buzzati (cfr. Lazzarin, 2005, p. 33, Buzzati, 1985, p. 248), in un contesto in cui il poeta discetta attorno alla teoria del piacere e in cui si riscontra la presenza del verbo "precipitare" e del sostantivo "precipizio": termini di scarsa frequenza nello Zibaldone, che, oltre a conferire il titolo, compaiono ben sette volte nella prosa buzzatiana:

Che cosa è la vita? Il viaggio di un zoppo e infermo che con un gravissimo carico in sul dosso per montagne ertissime e luoghi sommamente aspri, faticosi e difficili, alla neve, al gelo, alla pioggia, al vento, all'ardore del sole, cammina senza mai riposarsi dì e notte uno spazio di molte giornate per arrivare a un cotal precipizio o un fosso, e quivi inevitabilmente cadere. (Bologna, $17 \mathrm{Gen}$ 1826) (Leopardi, 1937-1938, vol. 2, p. 990).

In qualunque cosa tu non cerchi altro che piacere, tu non lo trovi mai: tu non provi altro che noia, e spesso disgusto. Bisogna, per provar piacere in qualunque azione ovvero occupazione, cercarvi qualche altro fine che il piacere stesso. (Può servire al Manuale di filosofia pratica). (30 Marzo 1827). [...] E forse per questa ragione gli spettacoli e i divertimenti pubblici per se stessi, senza altre circostanze, sono le più terribilmente noiose e fastidiose cose del mondo; perché non hanno altro fine che il piacere; questo solo vi si vuole, questo vi si aspetta; e una cosa da cui si aspetta e si esige piacere (come un debito) non ne dà quasi mai: dà anzi il contrario. Il piacere (si può dir con perfettissima verità) non vien mai se non inaspettato; e colà dove noi non lo cercavamo, non che lo sperassimo. Per questo nel bollore della gioventù, quando l'uomo si precipita col desiderio e colla speranza dietro al piacere, ei non prova che spaventevole e tormentoso disgusto e noia nelle più dilettevoli cose della vita. E non si comincia a provar qualche piacere nel mondo, se non sedato quell'impeto, e cominciata la freddezza, e ridotto l'uomo a curarsi poco e a disperare omai del piacere. (30 Marzo 1827; corsivi miei) (Leopardi, 1937-1938, vol. 2, pp. 1099-1100).

Il primo passo, giustamente celebre, è tradizionalmente utilizzato dagli esegeti per accompagnare il commento del Canto notturno di un pastore errante dell'Asia e collega intimamente l'idea del trascorrere del tempo a un crollo vertiginoso contro il quale gli esseri umani sono impotenti. Il secondo, invece, stabilisce un nesso stringente tra l'affanno della ricerca del piacere e l'età giovanile, quella cioè proverbialmente più avvinta al desiderio e alla speranza.

Se osservato in questa prospettiva intertestuale, l'ora che Marta si trova ad affrontare coincide con quella dell'"abisso orrido, immenso, / ov'ei 
precipitando, il tutto obblia" (Canto notturno di un pastore errante dell'Asia, vv. 35-36). Marta, come Nerina, trapassa velocemente ("Ma rapida passasti", Ricordanze, v. 152) e, durante il suo viaggio, si rende progressivamente ma ormai inutilmente conto dell'errore compiuto: "la paura di aver fatto uno sbaglio senza rimedio" (Buzzati, 2007, p. 309). Uno sbaglio che le fa perdere per sempre il bene a lei tanto caro, ossia proprio quella giovinezza che è la compagna di ogni desiderio, tanto è vero che al termine del racconto la ragazza è diventata "una vecchia decrepita". Allo stesso modo lo scrittore instaura un parallelismo tra l'arrivo della notte più fredda ("Sembrava notte profonda ormai", Buzzati, 2007, p. 309) e il tramonto definitivo della gioventù, sfruttando ancora la fonte leopardiana: "dopo il giorno sereno, / cadendo si dilegua, e par che dica / che la beata gioventù vien meno" (Il passero solitario, vv. 42-44).

E però con A Silvia che Ragazza che precipita mostra qualche ulteriore e più profonda tangenza. Entrambe le ragazze si collocano sul "limitare / di gioventù" (vv. 5-6), una soglia che Marta oltrepasserà gettandosi dal grattacielo e che Silvia supererà con la morte ("tua vita mortale", v. 2) che le impedirà di godere della vita. Entrambe le ragazze sono in attesa di una "festa": un'aspettativa che le lascerà deluse. Entrambe, infine, sono oggetto di attenzione da parte di spettatori che si rivolgono loro dai balconi ("d'in su i veroni del paterno ostello / porgea gli orecchi al suon della tua voce”, vv. 19-20). Ma è soprattutto nella chiusa - "All'apparir del vero / tu, misera, cadesti" (vv. 60-61) - che la comunanza fra i due testi assume maggiore e definitivo rilievo: se in Leopardi il crollo della speranza si consuma davanti al comparire della verità, nel racconto di Buzzati la ragazza che precipita cade; la sua morte non rappresenta solo la fine dell'esistenza, ma addita al venir meno di ogni speranza, consapevolezza che Marta assume proprio nel procedere della caduta:

Così sicura di sé quando aveva spiccato il volo, adesso Marta sentiva un tremito crescerle dentro, forse era semplicemente il freddo ma forse era anche paura, la paura di aver fatto uno sbaglio senza rimedio. [...]

Che ora era? All'ingresso del palazzo laggiù - che nel frattempo si era fatto più grande, e se ne potevano distinguere ormai tutti i particolari architettonici - permaneva intatta la luminaria, ma l'andirivieni delle automobili era cessato. Di quando in quando, anzi, piccoli gruppetti uscivano dal portone allontanandosi con passo stanco. Poi anche le lampade dell'ingresso si spensero. Marta sentì stringersi il cuore. Ahimè, alla festa, non sarebbe più giunta in tempo. Gettando un'occhiata all'insù, vide il pinnacolo del grattacielo in tutta la sua potenza crudele. Era quasi tutto buio, rare e sparse finestre ancora accese agli ultimi piani. E sopra la cima si spandeva lentamente il primo barlume dell'alba (Buzzati, 2007, pp. 309-310).

Marta è, dunque, non solo una "vecchia decrepita" ma addirittura "cadente" ("A questi piani bassi non passano che vecchie cadenti", Buzzati, 2007, p. 311), avverando la medesima immagine del "vecchierel" del Canto notturno che, come ribadito nello Zibaldone, giunge davanti al "precipizio" per "inevitabilmente cadere". La vecchiaia raggiunta da Marta è tale nell'aspetto, certo; 
ma lo è anche in profondità, nell'anima, di cui l'apparenza esteriore restituisce il riflesso, proprio a seguito della consapevolezza sopraggiunta nel percorso di caduta. Buzzati sfrutta qui il valore ambivalente all'aggettivo cadente: il termine è sinonimo, per traslato entrato nel linguaggio comune, di "decrepito, sfatto"; ma ha anche un genuino significato letterale, indicando appunto colei "che cade".

Nel 1962 Buzzati dipinge il quadro Ragazza che precipita, nel quale ricalca pari pari la vicenda della prosa pubblicata (e verosimilmente scritta) solo due anni prima. Si tratta di un dipinto, come spesso avviene in Buzzati, che incorpora frasi di testo, quasi fedelmente trapiantate dal racconto. Disponendo in sinossi le porzioni testuali presenti nel dipinto (colonna B) con quelle, ad esse coerenti, presenti nel racconto (colonna A), è possibile svolgere un immediato raffronto tra le opere: ${ }^{7}$

\begin{tabular}{|c|c|}
\hline A - Ragazza che precipita (racconto, & B - Ragazza che precipita (dipinto, 1962) \\
\hline & $\begin{array}{l}\text { MARTA } \\
\text { SPAVALDA SPAVALDA SARAI } \\
\text { SEMPRE TANTO SPAVALDA }\end{array}$ \\
\hline $\begin{array}{l}\text { vedendo di sotto la città risplendere } \\
\text { nella sera, fu presa dalle vertigini. [...] } \\
\text { un dolce abisso brulicante di luci; }\end{array}$ & $\begin{array}{l}\text { Vedendo di sotto la città risplendere } \\
\text { dolce abisso brulicante di luci FU } \\
\text { PRESA DALLE VERTIGINI }\end{array}$ \\
\hline $\begin{array}{l}\text { si sporse perdutamente oltre la } \\
\text { balaustra e si lasciò andare }\end{array}$ & si sporse perdutamente e si lasciò andare \\
\hline $\begin{array}{l}\text { Il sole, non ancora del tutto disceso, } \\
\text { fece del suo meglio per illuminare il } \\
\text { vestitino di Marta. Era un modesto } \\
\text { abito primaverile comprato-fatto } \\
\text { per pochi soldi. Ma la luce lirica } \\
\text { del tramonto lo esaltava alquanto, } \\
\text { rendendolo chic. }\end{array}$ & SI SENTIVA DI MODA \\
\hline $\begin{array}{l}\text { "Signorina, un piccolo drink?... } \\
\text { Gentile farfalla, perché non si ferma } \\
\text { un minuto tra noi?" } \\
\text { Lei rideva, svolazzando, felice } \\
\text { (ma intanto precipitava): "No, } \\
\text { grazie, amici. Non posso. Ho fretta } \\
\text { d'arrivare". } \\
\text { "Di arrivare dove?" le chiedevano. } \\
\text { "Ah, non fatemi parlare" rispondeva } \\
\text { Marta e agitava le mani in atto di } \\
\text { confidenziale saluto. }\end{array}$ & $\begin{array}{l}\text { Signorina, un piccolo drink? } \\
\text { Gentile farfalla, perché non si ferma un } \\
\text { minuto tra noi? } \\
\text { No grazie amici. Non posso. Ho fretta di } \\
\text { arrivare } \\
\text { Arrivare dove? } \\
\text { Ah, non fatemi parlare! }\end{array}$ \\
\hline
\end{tabular}

7. Nella tabella sono stati indicati in maiuscolo i segmenti testuali che nel quadro appaiono scritti in questo carattere. Nel dipinto le battute tra i personaggi non presentano virgolettature, per questo motivo non sono indicate in tabella. 


\begin{tabular}{|c|c|}
\hline "Come si permette, signore?" & Come si permette, signore? \\
\hline $\begin{array}{l}\text { "Lei ha tutta la vita davanti" le } \\
\text { dicevano "perché si affretta così? Ne } \\
\text { ha di tempo disponibile per correre e } \\
\text { affannarsi. Si fermi un momento con } \\
\text { noi, non è che una modesta festicciola } \\
\text { tra amici, intendiamoci, eppure si } \\
\text { troverà bene" }\end{array}$ & $\begin{array}{l}\text { Lei ha tutta la vita davanti perché si } \\
\text { affretta così? Si fermi un momento, una } \\
\text { piccola festicciola tra amici }\end{array}$ \\
\hline $\begin{array}{l}\text { come si precipita infatti allegramente } \\
\text { quando si hanno appena diciannove } \\
\text { anni. }\end{array}$ & come si precipita allegramente a quell'età \\
\hline $\begin{array}{l}\text { "Dove vai? Perché tanta fretta? } \\
\text { Chi sei?" le gridavano, nelle voci } \\
\text { si indovinava qualcosa di simile } \\
\text { all'invidia. } \\
\text { "Mi aspettano laggiù" rispondeva lei. } \\
\text { "Non posso fermarmi. Perdonatemi" }\end{array}$ & $\begin{array}{l}\text { DOVE VAI? PERCHÉ HAI TANTA } \\
\text { FRETTA? CHI SEI? } \\
\text { Mi aspettano laggiù non posso fermarmi }\end{array}$ \\
\hline $\begin{array}{l}\text { eccoci, siamo qui, è la nostra ora, } \\
\text { fateci festa, il mondo non è forse } \\
\text { nostro? }\end{array}$ & $\begin{array}{l}\text { eccoci, siamo qui è la nostra ora fateci } \\
\text { festa il mondo non è forse nostro? }\end{array}$ \\
\hline $\begin{array}{l}\text { "Alberto" gridò la moglie "hai visto? È } \\
\text { passata una donna". } \\
\text { "Com'era?" fece lui senza alzare gli } \\
\text { occhi dal giornale. } \\
\text { "Una vecchia" rispose la moglie } \\
\text { "Una vecchia decrepita. Sembrava } \\
\text { spaventata". } \\
\text { "Sempre cosi" l'uomo brontolò. } \\
\text { "A questi piani bassi non passano } \\
\text { che vecchie cadenti. Belle ragazze } \\
\text { si vedono dal cinquecentesimo } \\
\text { piano in su. Mica per niente quegli } \\
\text { appartamenti costano così cari". } \\
\text { "C’è il vantaggio" osservò la moglie } \\
\text { "che quaggiù almeno si può sentire il } \\
\text { tonfo, quando toccano terra". } \\
\text { "Stavolta, neanche quello" disse lui, } \\
\text { scuotendo il capo, dopo essere rimasto } \\
\text { alcuni istanti in ascolto. E bevve un } \\
\text { altro sorso di caffe. }\end{array}$ & $\begin{array}{l}\text { Alberto, hai visto? è passata una } \\
\text { Una cosa? } \\
\text { Una... come dire? una specie di stella, } \\
\text { proprio una minuscola stellina. } \\
\text { AH! }\end{array}$ \\
\hline
\end{tabular}

Le frazioni testuali presenti nel dipinto ricalcano in buona misura i relativi passaggi del racconto di cui offrono spesso una variante improntata alla sintesi a motivo della evidente differenza di supporto. Inoltre, alcune frasi o parole appaiono scritte nel dipinto in carattere maiuscolo, con l'intendimento di conferire maggiore enfasi a battute o a passaggi. 
Al di là delle evidenti somiglianze, vi sono però differenze altrettanto notevoli che insistono in particolare al principio e alla conclusione della storia dipinta, mentre lo svolgimento della narrazione, con la caduta progressiva di Marta, è improntato a una sostanziale omogeneità. Nella soglia iniziale Buzzati inserisce un'immagine, una sorta di "quadro nel quadro" che ritrae Marta per tutta l'altezza del dipinto, ottenendo una figura assolutamente sproporzionata rispetto alla seconda sequenza di carattere più spiccatamente narrativo che propone la caduta della ragazza parallelamente alla parete del grattacielo. Nella prima scena, la figura di Marta risulta in tal modo esageratamente ingigantita; inoltre la sua stessa postura ritta ed impettita e lo sguardo intenso e fiero conferiscono alla ragazza un marcato carattere di sfrontatezza reso manifesto dalle parole maiuscole che accompagnano il personaggio ("Spavalda spavalda sarai sempre tanto spavalda?”). Altra, e sostanziale, differenza con la prosa è la conclusione che presenta, anche qui, un dialogo all'interno di un appartamento; Marta però non è più assimilata a una "vecchia" ma a una "minuscola stellina". A riaffermare la differenza fra le due opere, infine, se nel racconto l'uomo lamenta il fatto di non essere riuscito a sentire il "tonfo" di colei che precipita, nel dipinto la conclusione della storia è lasciata al grido di dolore di Marta che si schianta al suolo (“AH!”). Questa conclusione grafica è collocata certamente in prossimità del punto di caduta, ma in posizione più discosta se raffrontata ad altre battute del dipinto decisamente più addossate ai diversi personaggi e può consentire una lettura ulteriore. La particella grammaticale $a h$ ! è un'interiezione che può essere, come è noto, utilizzata con una vasta gamma di sfumature, dal dolore alla sorpresa, dal desiderio al riso. In quest'ottica la conclusione sfrutta la particolare intonazione emotiva del parlante, che si trasferisce su chi osserva/legge il dipinto e non può non collegare l'attacco di questa storia con la sua fine. Per cui si avrà una domanda iniziale ("Spavalda spavalda sarai sempre tanto spavalda?”) e una risposta conclusiva: “AH!”. Tale epilogo offre una replica alla questione introduttiva per cui Marta sarà spavalda finché non cadrà, ovvero non morirà, quasi a ribadire un tema ricorrente nella tradizione letteraria (da Dante a Shakespeare e oltre) e rinfocolato dalla lettura di scapigliati e simbolisti relativo alla donna superba, la cui altezzosa bellezza viene inesorabilmente cancellata dallo scorrere del tempo.

Con l'inserzione della sequenza d'esordio e con il cambiamento della conclusione, il dipinto sembra mutare radicalmente non tanto l'impianto narrativo quanto piuttosto il nucleo argomentativo di Ragazza che precipita, trapassando da una forma di indagine sull'esistenza alla rielaborata immagine di un esempio di tracotanza punita. A questo stravolgimento tematico può con molta probabilità aver contribuito la scrittura di Un amore, in fase di stesura proprio durante la realizzazione di questo dipinto. Il romanzo, che come è noto ha salde radici nella biografia dell'uomo Buzzati, narra l'ossessione di Antonio Dorigo per la giovane Laide, irraggiungibile e lontana nel sentimento quanto invece è disponibile nel commercio carnale. ${ }^{8}$ Il dipinto

8. Risale al 1959 l'incontro con la giovane donna, nota con le iniziali di S.C., che diventerà 
non si configura quindi come semplice rifacimento o interpretazione grafica della precedente prosa, ma impone uno slittamento tematico. Buzzati inventa a tutti gli effetti un'altra opera, capace di richiamare il testo del racconto ma al contempo in grado di entrare in frizione con esso lasciando emergere valori nuovi, segno di un'ispirazione fecondissima, sapiente nel narrare e continuamente riplasmare le proprie storie, anche quando esse presuppongono un cambiamento di linguaggio espressivo.

\section{Bibliografia}

Blasucci, L. (1985). Leopardi e i segnali dell'infinito. Bologna: Il Mulino.

Boehm, G. (2009). La svolta iconica (a cura di M.G. \& M. Di Monte). Roma: Meltemi.

Buzzati, D. (1958). Un equivoco. In Le storie dipinte. Milano: All'insegna dei Re Magi. Buzzati, D. (1960, 16 marzo). Ragazza che precipita. Corriere della Sera, p. 3.

Buzzati, D. (1963). Un amore. Milano: Mondadori.

Buzzati, D. (1966). Il colombre. Milano: Mondadori.

Buzzati, D. (1968). La boutique del mistero. Milano: Mondadori.

Buzzati, D. (1971). Dino Buzzati in un "incontro" condotto dal critico letterario Ferdinando Giannessi. I Quaderni de L'argine, 3.

Buzzati, D. (1985). Lettere a Brambilla (a cura di L. Simonelli). Novara: De Agostini. Buzzati, D. (2006). I sette messaggeri. Milano: Mondadori. (1a ed. 1942).

Buzzati, D. (2007). Il colombre. Milano: Mondadori. (1a ed. 1966).

Buzzati, D. (2010). I fuorilegge della montagna. Uomini, cime, imprese (a cura di L. Viganò). Milano: Mondadori.

Buzzati, D. (2013). Le storie dipinte (a cura di L. Viganò). Milano: Mondadori.

Buzzati, D. (2018). Il reggimento parte all'alba (a cura di L. Viganò). Milano: Henry Beyle.

Caspar, M.-H. (1979). Les Miracles de Val Morel: un bestiaire fantastique?, Cahiers Buzzati, 3, pp. 140-172.

Caspar, M.-H. (2000). A propos du paratexte buzzatien, Studi buzzatiani, 5, pp. 27-46. Cavadini, L. (Ed.) (2001). Parole e colori. Cernobbio: Comune di Cernobbio.

Cometa, M. (2004). Parole che dipingono. Roma: Meltemi.

Cometa, M. (2012). La scrittura delle immagini. Letteratura e cultura visuale. Milano: Cortina.

Crotti, I. (1977). Dino Buzzati. Firenze: La Nuova Italia.

Dal Mas, S., \& Endrizzi, S. (1998). La 'Spiegazione' ai Miracoli di Val Morel come racconto di un viaggio all'altro mondo. Studi buzzatiani, 3, pp. 133-142.

Ferrari, M.T. (Ed.) (2006). Buzzati racconta. Storie disegnate e dipinte. Milano: Electa. Giannetto, N. (1998). L'icona parlante: intercambiabilità e complementarità di parola e immagine nel Buzzati pittore e disegnatore. In M. Ciccuto \& A. Zingone (Edd.), I segni incrociati. Letteratura italiana del '900 e arte figurativa (pp. 585-602). Lucca: Mauro Baroni.

Giannetto, N. (2001). La parola scritta nei dipinti e nei disegni di Buzzati. In Cavadini (Ed.) (2001), pp. 17-25.

la protagonista di Un amore. Sulla questione si vedano Monelli (1965, p. 111) e Buzzati (2010, p. LIX-LX). 
Laganà Gion, A. (1982). Caratteri unitari nell'opera di Buzzati: i rapporti tra letteratura e pittura. In A. Fontanella (Ed.). Dino Buzzati (pp. 291-311). Firenze: Olschki.

Lazzarin, S. (2005). "Le immense cose che si erano sognate"... Costanti evocative e presenze leopardiane nella narrativa breve di Buzzati. Italianistica, 34 (1), pp. 33-47.

Leopardi, G. (1937-1938). Zibaldone di pensieri (a cura di F. Flora). Milano: Mondadori.

Monelli, P. (1965). Ombre cinesi. Scrittori al girarrosto. Milano: Mondadori.

Mitchell, W.J.T. (1994). Picture Theory. Essays on Verbal and Visual Representation. Chicago-London: The University of Chicago Press.

Mitchell, W.J.T. (2017). Pictorial turn. Saggi di cultura visuale (a cura di M. Cometa \& V. Cammarata). Milano: Raffaello Cortina.

Mitchell, W.J.T. (2018). Scienza delle immagini. Iconologia, cultura visuale ed estetica dei media. Monza: Johan\&Levi.

Piccolo, F. (2011). Segni e di-segni. Storia de "La famosa invasione degli orsi in Sicilia" di Dino Buzzati. In I. Crotti, E. Del Tedesco, R. Ricorda \& A. Zava (Edd.), Autori, lettori e mercato nella modernità letteraria (Vol. 1, pp. 634-638). Pisa: ETS.

Sandrini, G. (2001). Presenza di Leopardi nel primo Buzzati. Studi buzzatiani, 6, pp. 7-19.

Spigoli, T. (Ed.) (2018). Verba picta. Interrelazione tra testo e immagine nel patrimonio artistico e letterario della seconda metà del Novecento. Pisa: ETS.

Toscani, C. (1987). Guida alla lettura di Buzzati. Milano: Mondadori.

Viganò, L. (2006). L'unico vero pittore del XX secolo. In Ferrari (Ed.) (2006), pp. $17-19$.

Zucco, M.E. (1997). Fonti iconografiche della pittura di Buzzati. Studi buzzatiani, 2, pp. 34-71. 


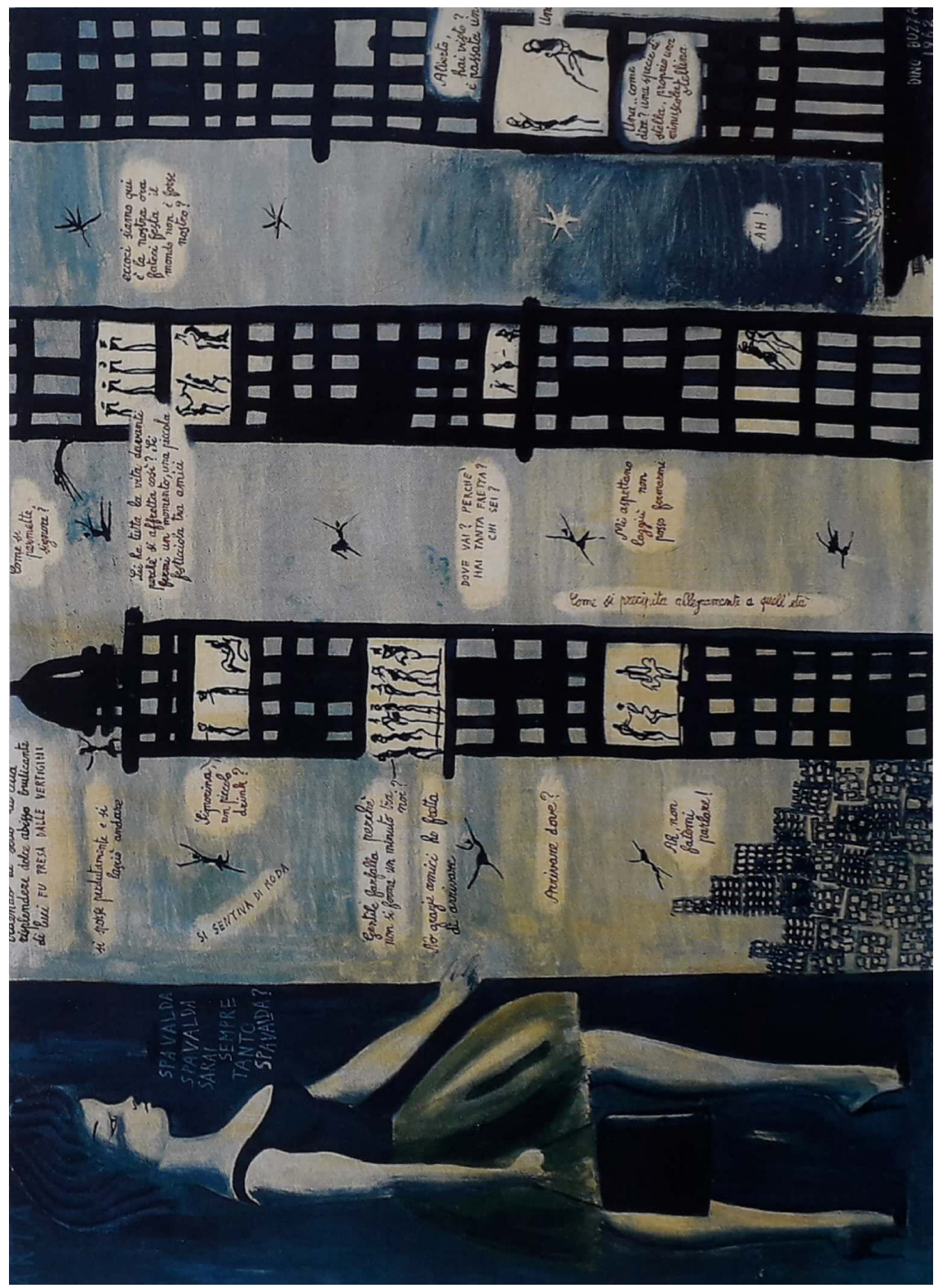

Dino Buzzati, Ragazza che precipita, 1962, tempera su tela, 70×100 cm (collezione privata). 
